\title{
CONSORT, QUOROM, and structured abstracts - new rules for authors, new tools for readers
}

There is no innovation in clinical medicine without the publication of research results. Data from research, however, have to be unbiased, easily available, completely reported, and comprehensible. Much has been done in recent years to decrease bias in clinical trials and to increase availability of research data. For instance, it has now been widely accepted that the gold-standard study design to establish an intervention's efficacy and its absence of harm is the controlled randomized double-blind trial. Although not everything can be reported in a randomized trial [1], empirical evidence strongly suggests that this study design ensures minimization of selection bias and observer bias, both of which may lead to the overestimation of the effect of a treatment [2]. There is no reason to assume that this wisdom should not be applicable to clinical trials in perioperative medicine [3].

The most valid randomized trial, however, is of no use, if the results of that trial are reported in an incomprehensible fashion or incompletely. In order to improve this, an international group of people, statisticians, editors and epidemiologists, met in 1995. The result of this collaborative work was the Consolidated Standards of Reporting Trials (CONSORT) statement [4,5]. The main requirement of the statement is that authors should provide enough information for readers to know how the trial was performed so that they can judge whether the findings are reliable. The statement contains a list of items that must be checked by the authors before submission of their paper. For instance, authors will have to declare the method of randomization they have used. The statement also suggests the use of structured abstracts; subheadings (as opposed to a one-paragraph text) have been recommended to increase the quality and content of abstracts [6]. Finally, the CONSORT statement proposes the graphical display of a flow diagram, which allows both peer-reviewers and readers to follow the progress of participants through the trial.

Increasingly, systematic reviews of randomized trials are published in the medical literature, and undoubtedly their impact on medical decision making is constantly rising. This also applies to the literature on perioperative medicine [7]. However, as with randomized trials, the quality of reporting of data from qualitative and quantitative systematic reviews (i.e. meta-analyses) must be ensured. With the aim to improve the reporting of these reports, yet another international group has published the Quality of Reporting of Meta-Analyses (QUOROM) statement [8]. As with the CONSORT statement, there is a checklist, which includes, for instance, the recommendation to use structured abstracts, to describe in detail the literature search, and to provide a diagram that displays the flow of the retrieved reports. And as with the CONSORT statement, the aim is to increase completeness of reporting of these studies, and to help improve their design and conduct.

In August 2000, of 15 locally available international Anaesthesia and Pain journals, six (Annales Françaises d'Anesthésie et de Réanimation, Anesthesiology, Acta Anaesthesiologica Scandinavica, Canadian Journal of Anaesthesia, Der Anästhesist, Journal of Clinical Anesthesia, and Regional Anesthesia and Pain Management) requested a structured abstract in their Guide for Authors. None referred to the CONSORT or the QUOROM statement. The Editors of the European Journal of Anaesthesiology have decided to endorse the use of structured abstracts, despite some open questions [9]. The Journal will even go a step further. From 1 January 2001, authors of clinical trials will be asked to refer to the CONSORT statement, and authors of systematic reviews to the QUOROM statement. Authors of these studies will be asked to include a flow diagram of participants (clinical trial) and retrieved reports (systematic review). This will facilitate peer-review and help readers to judge the progress of data through a publication and its completeness. The aim is to ensure the highest quality and validity of original contributions in the Journal and to further increase its scientific impact. The Editors of the European Journal of Anaesthesiology hope that these new rules for authors 
will become appreciated tools for peer-reviewers and readers.

\section{Martin R. Tramèr \\ Division of Anaesthesiology, \\ Department APSIC, \\ Geneva University Hospitals, \\ CH-1211 Geneva 14, Switzerland}

\section{References}

1 Black N. Why we need observational studies to evaluate the effectiveness of health care. BMJ 1996; $\mathbf{3 1 2}$ : 1215-1218.

2 Schulz KF, Chalmers I, Hayes RJ, Altman DG. Empirical evidence of bias. Dimensions of methodological quality associated with estimates of treatment in controlled trials. JAMA 1995; 273: 408-412.

3 Carroll D, Tramèr M, McQuay H, Nye B, Moore A. Randomization is important in studies with pain outcomes: systematic review of transcutaneous electrical nerve stimulation in acute postoperative pain. Br J Anaesth 1996; 77: 798-803.

4 Begg C, Cho M, Eastwood S et al. Improving the quality of reporting of randomized controlled trials. The CONSORT Statement. JAMA 1996; 276: 637-639.

5 Altman DG. Better reporting of randomised controlled trials: the CONSORT statement. BMJ 1996; 313: 570-571.

6 Taddio A, Pain T, Fassos FF, Boon H, llersich AL, Einarson TR. Quality of nonstructured and structured abstracts of original research articles in the British Medical Journal, the Canadian Medical Association Journal and the Journal of the American Medical Association. CMAJ 1994; 150: 1611-1615.

7 http://www.hcuge.ch/anesthesie/anglais/evidence/arevusyst. htm

8 Moher D, Cook DJ, Eastwood S et al. Improving the quality of reports of meta-analyses of randomised controlled trials: the QUOROM statement. Quality of reporting of meta-analyses. Lancet 1999; 354: 1896-1900.

9 Scherer RW, Crawley B. Reporting of randomized clinical trial descriptors and use of structured abstracts. JAMA 1998; 280: 269-272.

(C) 2001 European Academy of Anaesthesiology, European Journal of Anaesthesiology, 18, 1-2 